\title{
Thomas Rahn, Hole Rößler (dir.), Medienphantasie und Medienreflexion in der Frühen Neuzeit
}

\section{Claire Gantet}

\section{OpenEdition}

\section{Journals}

Édition électronique

URL : http://journals.openedition.org/ifha/10274

ISSN : 2198-8943

\section{Éditeur}

IFRA - Institut franco-allemand (sciences historiques et sociales)

Référence électronique

Claire Gantet, «Thomas Rahn, Hole Rößler (dir.), Medienphantasie und Medienreflexion in der Frühen Neuzeit », Revue de l'IFHA [En ligne], Date de recension, mis en ligne le 08 août 2019, consulté le 23 septembre 2020. URL : http://journals.openedition.org/ifha/10274

Ce document a été généré automatiquement le 23 septembre 2020.

(CIFHA 
Thomas Rahn, Hole Rößler (dir.), Medienphantasie und Medienreflexion in der Frühen Neuzeit

\author{
Claire Gantet
}

\title{
RÉFÉRENCE
}

Thomas Rahn, Hole Rößler (dir.), Medienphantasie und Medienreflexion in der Frühen Neuzeit. Festschrift für Jörg Jochen Berns, Wiesbaden : Harrassowitz Verlag in Kommission (Wolfenbütteler Forschungen, 157), 2018, 419 p., $82 €$ 
De Jörg Jochen Berns, on a dit qu'il était connu comme «le loup blanc des études allemandes sur le baroque». Ses recherches ont porté sur la littérature baroque, l'histoire de la germanistique, les journaux, le cérémoniel, la mythographie, la mnémonique, les images et techniques visuelles (le film avant le film): sur les textes ancrés dans d'autres médias. Cela justifie l'édition de mélanges pour son quatre-vingtième anniversaire, un rare honneur - et une recension par une nongermaniste non spécialiste du baroque et lectrice de ses travaux. L'ouvrage consacré à Jörg Jochen Berns sera donc lu au prisme de ses suggestions au regard de la question historienne du statut et des langages des documents pris comme

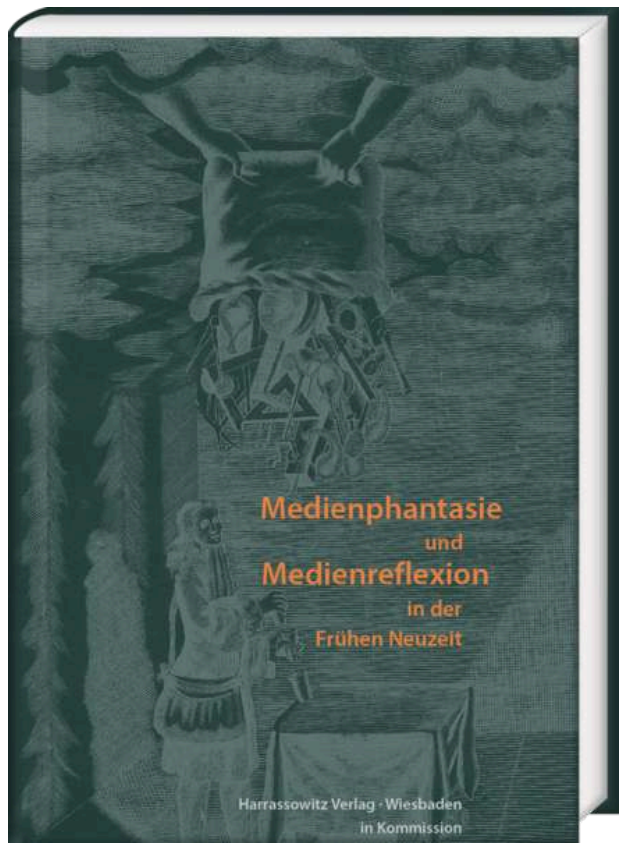
sources.

Une stimulante introduction par Thomas Rahn et Hole Rößler situe l'œuvre de Jörg Jochen Berns : une «théologie des médias » opposée au déterminisme de Marshall McLuhan («le média, c'est le message!»), attentive à une historicisation précise, à l'appropriation des techniques et leur intériorisation productive par l'imaginatio et la memoria. Les médias dissipaient, apaisaient, mais pouvaient aussi concentrer l'attention du chrétien et devenir un objet de méditation et de poésie pieuse, voire servir de métaphores de l'âme humaine. La dimension technique des médias portait un effet de distanciation, soutenait un scepticisme et une réflexion sur leurs incidences en même temps qu'elle stimulait la création littéraire. Loin d'être le propre de l'ère des mass media, certains effets - ainsi le recouvrement de l'information par le divertissement étaient ainsi déjà au XVII ${ }^{e}$ siècle le propre de la littérature de curiosité et des romans, qui véhiculaient aussi des savoirs. Les journaux, une nouveauté du $\mathrm{XVII}^{\mathrm{e}}$ siècle, promettaient étrangeté et nouveauté - et par là caressaient les affects (plaisir et peur, répulsion et lassitude) - en même temps qu'ils accéléraient la communication. Les images furent concomitamment éditées en nombre croissant; la violence à l'encontre des images en fut peut-être une conséquence.

Une première partie envisage les réflexions sur les médias. La duplication précaire par l'imprimerie alla de pair avec une dévalorisation du terme copia (richesse, abondance) en copie, et l'écho (une nymphe des Métamorphoses d'Ovide, flanquée de Narcisse), avec son caractère laconique, oraculaire et désespérant, thématisa poétiquement le rapport aux médias (Anita Traninger). La reproduction massive par l'imprimerie affecta aussi le statut de l'image, si bien qu'émergèrent des stratégies et tactiques propres à transformer des portraits en médias de diffamation, avant l'âge de la caricature (Hole Rößler). Le spectacle théâtral reposait sur l'usage de machines en dialogue avec les textes, à la croisée d'ères temporelles et d'aires géographiques (Jan Lazardzig). La valeur épistémique de l'image fut forte, en particulier dans le cas de la généalogie, où la figure de l'arbre obligeait à représenter l'origine souvent nébuleuse de telle famille 
(dans les racines) et à simplifier son histoire aux dépens des branches collatérales ou d'une période, en s'accompagnant parfois de textes bucoliques (Volker Bauer).

La deuxième partie s'attache à la transmission par les médias : ainsi, par exemple, un poème épique resté dans l'oubli sur la mort du duc Magnus de Wurtemberg lors de la bataille de Wimpfen en 1622 (Wilhelm Kühlmann), ou les lettres personnelles - et chiffrées - de la Palatine (Liselotte von der Pfalz empreinte de son éducation calviniste) ou de la catholique Marie-Amélie de Saxe, affligées de chagrin comme lors du décès d'un enfant (Helen Watanabe-O'Kelly).

Une troisième partie se penche sur les expériences sur les médias et leur imaginaire. Wolfgang Brückle pose la question de l'épineuse définition de la photographie et propose une typologie des images du début du XVII ${ }^{e}$ siècle qui se voulaient proches de la réalité. Les images d'explosion sont, quant à elles, à contextualiser dans plusieurs registres : enregistrement des dégâts, effroi, recours à la fiction (en raison de leur caractère éphémère et naturel), emprunts au répertoire du feu d'artifice aulique, dimension politique du retour à l'ordre urbain (Thomas Rahn). Au moyen d'images, Joseph Imorde retrace comment les savants et les visiteurs du XVIII siècle se reconstituaient sur place la Rome antique au moyen des ruines et de façon animée, par exemple de nuit. Helga Meise étudie l'Almanach domestique (1782) de Caroline von Keyserling, resté inconnu jusque peu, qui subvertit le genre des almanach et des almanachs des muses : il s'agit d'un document écrit en français et dessiné à la main par la comtesse, mêlant poésie bucolique, cérémoniel aulique, soins de santé (le fameux trémoussoir), sociabilité des Lumières ainsi qu'un idéal de vie et une certaine ironie.

Les transformations de l'être humain et des médias constituent le thème suivant. C'est sur l'iconographie du "moulin mystique» représentant l'offrande eucharistique comme un "automate sacré " (Jörg Jochen Berns) et son évolution que se penche Christian Hecht. Dès l'Antiquité, l'écriture poétique fut mise en relation avec des processus corporels. Günter Butzer analyse brillamment les métaphores de la bibliophagie notamment chez Montaigne, les troubles de la digestion affectant l'imagination; à la différence de l'ingestion de matériaux du monde externe, l'image interne du sang est riche de connotations identitaires (le siège de l'âme) et religieuses (le sang du Christ et la communauté des chrétiens) unidirectionnelles asymétriques; attachées à la compassion du Christ, les larmes, au XVII et plus encore au XVIII ${ }^{\mathrm{e}}$ siècle sont aussi versées en contexte laïc et ont une vertu communicative multidirectionnelle : il existe une " poétique physiologique ».

L'ultime partie est une gravure d'Ad Stijnman intitulée Esthétique des médias et accompagnée d'un poème.

À la lecture de ce volume, on regrettera seulement qu'il n'ait guère traité du thème stimulant des «machines de l'âme ». Il n'empêche : il documente les renouvellements foisonnants des questionnements de la germanistique, à la croisée de l'étude des textes, des images, des médias, des machines et des corps. C'est une lecture riche et vivifiante. 
INDEX

Thèmes : Histoire de la culture

Index chronologique : Période moderne

\section{AUTEURS}

\section{CLAIRE GANTET}

Université de Fribourg (Suisse)/Universität Freiburg (Schweiz) 